\title{
Determinação da Distância de Dispersão Efetiva de Cabralea canjerana
}

\author{
Anna Paula Lora Zimmermann ${ }^{1}$, Frederico Dimas Fleig ${ }^{1}$, \\ Gabriel Paes Marangon ${ }^{1}$ \\ ${ }^{1}$ Centro de Ciências Rurais, Universidade Federal de Santa Maria - UFSM, Santa Maria/RS, Brasil
}

\begin{abstract}
RESUMO
Objetivou-se conhecer a distância de dispersão efetiva de Cabralea canjerana (Vell.) Mart. em um fragmento de Floresta Estacional. Foram alocadas parcelas de $5 \mathrm{~m} \times 5 \mathrm{~m}$ em uma área com alta densidade de indivíduos regenerantes da espécie. Os indivíduos com altura maior que $30 \mathrm{~cm}$ e DAP menor que $15 \mathrm{~cm}$ foram contabilizados e tiveram suas coordenadas relativas conhecidas. Indivíduos com DAP maior que $15 \mathrm{~cm}$ foram considerados como produtores de sementes. A distância de dispersão foi calculada através da metodologia de Nathan \& Muller-Landau (2000), sendo calculadas duas distâncias: distância de cada indivíduo em relação à matriz mais próxima e em relação à média das distâncias entre todas as matrizes. O pico de dispersão efetiva ocorre a uma distância média de 30 metros das árvores matrizes, sendo necessário três indivíduos produtores de sementes por hectare para se garantir a regeneração contínua da espécie.
\end{abstract}

Palavras-chave: dispersão de sementes, regeneração natural, densidade de sementes.

\section{Determination of the Effective Dispersal Distance of Cabralea canjerana}

\begin{abstract}
This study aimed to know the effective dispersal distance of Cabralea canjerana (Vell.) Mart. in a forest fragment. Plots of $5 \times 5 \mathrm{~m}$ were allocated in an area with high density of regenerating individuals of the species. Individuals higher than $30 \mathrm{~cm}$ and with diameter at breast height (DBH) smaller than $15 \mathrm{~cm}$ were recorded and referenced in pseudo-coordinates. Individuals with DBH greater than $15 \mathrm{~cm}$ were considered as seed producers. The dispersal distance was calculated using the methodology by Nathan \& Muller-Landau (2000); two distances were calculated: the distance of each individual in relation to the nearest adult tree, and the average distance between all matrix trees. The effective dispersal peak occurs at a mean distance of 30 meters from the mother trees. Three seed producing individuals per hectare are needed to ensure the continuous regeneration of the species.
\end{abstract}

Keywords: seed dispersal, natural regeneration, seed density. 


\section{INTRODUÇÃO}

O processo de dispersão é complexo e envolve relações específicas entre plantas e dispersores. Assim, os diásporos evoluíram ao longo do tempo e adaptaram-se para facilitar sua dispersão. Sementes dispersas zoocoricamente geralmente possuem polpas comestíveis, são doces e coloridas, mostrando-se atraentes a diversos animais, como por exemplo, às aves (Sork, 1987). Dentre os exemplos de mutualismo entre plantas e dispersores, a principal recompensa dada pelas plantas aos frugívoros parece ser o retorno nutricional dado pela ingestão da polpa dos frutos (Rocha et al., 2004). Em estudo realizado com seis espécies florestais, Pizo \& Oliveira (2001), Cabralea canjerana (Vell.) Mart. apresentou os maiores teores de lipídios e proteínas, demonstrando sua rentabilidade energética.

O tamanho da semente também exerce influência no processo de dispersão, assim como o hábito do dispersor, os quais determinam a distância de dispersão que a semente pode alcançar. Sementes grandes são mais difíceis de serem dispersas do que as pequenas, por não poderem ser carregadas a longas distâncias e por necessitarem de animais de maior porte (Janzen, 1970).

Conhecer a distância de dispersão das sementes de espécies florestais de interesse pode ser uma ferramenta útil ao processo de sucessão, principalmente em áreas degradadas, onde a regeneração natural deve ser novamente estabelecida. Diversos trabalhos, como os de Donatti (2004), Rabello et al. (2010), Lopes et al. (2010), Nathan \& Muller-Landau (2000), também buscaram conhecer a distância de dispersão de sementes. A partir da determinação da distância que as sementes conseguem percorrer é possível se manejar a área através do sistema de árvores porta-sementes fornecendo diásporos de forma eficiente para que haja o recobrimento do local e contribuindo assim para a recuperação de ecossistemas, manutenção de áreas em equilíbrio e perpetuação de florestas.

Cabralea canjerana é uma das espécies mais importantes encontradas na Floresta Estacional Decidual do sul do Brasil (Hack et al., 2005). Por suas características satisfatórias e amplo uso na construção civil e movelaria, essa espécie sofreu grande exploração no passado, tendo sua população reduzida e sendo considerada, hoje, como espécie vulnerável à extinção (Ferreira et al., 2005).
As sementes de C. canjerana medem de $6 \mathrm{~mm}$ a $17 \mathrm{~mm}$ e apresentam arilo suculento e atrativo de cor alaranjada, macio e carnoso, sendo ela dispersa quase que exclusivamente por pássaros (Pizo, 1995; Carvalho, 2003). Não apresentam dormência mas são altamente recalcitrantes, sendo intolerantes à perda de água e perdendo sua viabilidade em pouco tempo, além de serem altamente depredadas por insetos e roedores (Pizo, 1995).

Diante disso, este trabalho objetivou conhecer a distância de dispersão efetiva de sementes de C. canjerana em uma área de fragmento de Floresta Estacional Decidual, a fim de contribuir para o manejo e perpetuação da espécie.

\section{MATERIAL E MÉTODO}

O estudo foi realizado em um fragmento de Floresta Estacional Decidual no município de Silveira Martins, Região Central do Rio Grande do Sul. A fisionomia dessa formação florestal caracteriza-se por apresentar estrato superior caducifólio, no qual mais de $50 \%$ dos indivíduos perdem suas folhas no período desfavorável ao crescimento (IBGE, 2012). O clima da região, segundo a classificação de Köppen, é do tipo $\mathrm{Cfa}$, caracterizado como subtropical úmido, podendo chegar até $1.700 \mathrm{~mm}$ de precipitação anual. Mesmo não havendo uma estação seca definida, pode ocorrer déficit hídrico, principalmente nos meses mais quentes do ano (Heldwein et al., 2009). Os solos predominantes são do tipo Neossolo Litólico Húmico típico, pouco profundos e bem drenados (Embrapa, 2006).

Para os estudos da distância de dispersão efetiva de sementes de $C$. canjerana e avaliação no fragmento foram alocadas 144 parcelas contíguas de $25 \mathrm{~m}^{2}(5 \mathrm{~m} \times 5 \mathrm{~m})$ no local, totalizando $3.600 \mathrm{~m}^{2}$ de área amostrada. Todos os indivíduos da espécie com altura igual ou superior a $30 \mathrm{~cm}$ e diâmetro à altura do peito inferior a $15 \mathrm{~cm}$ que continham fustes inclusos dentro dos limites das parcelas foram contabilizados. O critério de inclusão acima de $30 \mathrm{~cm}$ foi baseado no fato de que plântulas menores que o limite proposto poderiam apresentar dificuldades de visualização que poderiam acarretar no seu pisoteamento, além de isso comprometer a identificação correta da espécie, uma vez que quando jovem a plântula de C. canjerana assemelha-se a Cedrela fissilis Vell. 
A localização dos indivíduos nas parcelas foi baseada no sistema de coordenadas relativas, no qual o vértice inferior direito de cada parcela foi tomado sempre como referência (ponto 0,0). Uma bússola acoplada a um tripé foi ajustada no ponto de referência e foi realizada a leitura da distância do vértice até a árvore e o ângulo formado por ela e o eixo da parcela (Figura 1). A transformação desses valores foi realizada em planilha Excel, por meio da multiplicação pelo seno e cosseno do ângulo, determinando a posição de cada indivíduo dentro da parcela.

A partir das coordenadas de cada árvore dentro da parcela, foi possível calcular a distância entre os indivíduos de regeneração natural e as árvores matrizes selecionadas, a partir da metodologia proposta por Nathan \& Muller-Landau (2000). Os indivíduos de C. canjerana com diâmetro a altura do peito maior ou igual a $15 \mathrm{~cm}$ foram considerados árvores produtoras de sementes e também tiveram suas coordenadas relativas conhecidas.

Calculadas as distâncias de dispersão mínima e média para cada indivíduo em relação a cada árvore matriz, estimou-se o número de plantas nas classes de distância, que variaram entre 0 e 55 metros, com intervalos de 5 metros. Foram plotados gráficos para análise da distância de dispersão efetiva da C. canjerana, considerando a distância de cada ponto em relação à matriz mais próxima e em relação à média das distâncias entre todas as matrizes.

\section{RESULTADOS E DISCUSSÃO}

Foram analisados 86 indivíduos na classe de regeneração natural e cinco indivíduos com diâmetro à altura do peito maior ou igual a $15 \mathrm{~cm}$, de acordo com o limite proposto para ser considerada possível árvore dispersora de sementes no local (Figura 2).

Considerando que cada indivíduo regenerante de C. canjerana presente na área é oriundo da árvore matriz mais próxima a ele, a distância mínima entre ele e a árvore produtora de sementes foi de 3,6 metros, estando localizado possivelmente sob a copa da árvore matriz. A distância mínima média, considerando que cada indivíduo de regeneração tem a probabilidade de ser oriundo de qualquer uma das cinco árvores matrizes da área foi de 13,2 metros. Entretanto, ao se observar a distância média de dispersão, nota-se que a maioria dos indivíduos regenerantes de canjerana encontram-se dispersos num intervalo entre 19,6 m e 28,6 m, respectivamente para a possibilidade de que a semente tenha vindo da matriz mais próxima ou de qualquer árvore matriz da área (Tabela 1).

A teoria proposta por Janzen (1970) e Connell (1971) sugere que há uma proporcionalidade entre a densidade e a distância das sementes à planta mãe. Assim, quanto mais próximo da árvore matriz, maior a predação de sementes. Isso ocorre pois os patógenos da árvore matriz acabam tornando-se predadores das sementes dispersas sob sua copa. Além disso, como a densidade de sementes é maior próxima a matriz, há

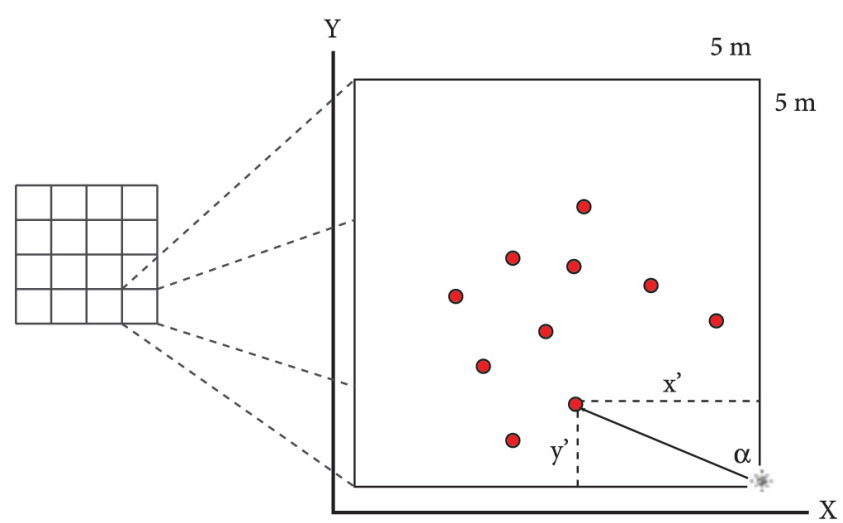

Figura 1. Esquema para obtenção das coordenadas relativas (x’, y') de cada indivíduo de Cabralea canjerana para o estudo da dispersão efetiva de sementes.

Figure 1. Scheme for obtaining the relative coordinates (x', y') of each individual Cabralea canjerana to study the effective dispersion of the seeds. 


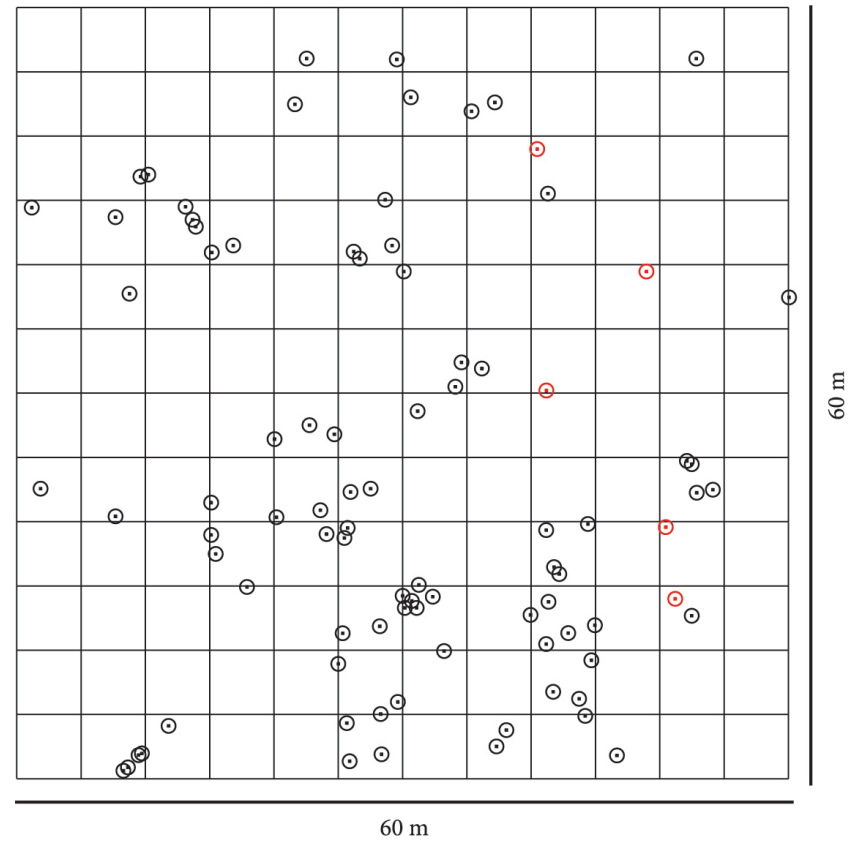

Figura 2. Distribuição espacial de indivíduos regenerantes e árvores matrizes (destacadas em vermelho) de Cabralea canjerana em fragmento de Floresta Estacional Decidual, RS.

Figure 2. Spatial distribution of regenerating and matrices tree (highlighted in red) of the Cabralea canjerana in fragment of the Deciduous Forest, RS.

Tabela 1. Distâncias de dispersão mínima e média, calculadas entre os indivíduos regenerantes e as árvores matrizes do local de estudo.

Table 1. Dispersal distance minimum and average, calculated between natural regeneration and the matrices trees in study site.

\begin{tabular}{lccccccc}
\multicolumn{1}{c}{ Indivíduo } & \multicolumn{9}{c}{ Matriz } & Distância mínima & Distância média \\
\cline { 2 - 7 } & $\mathbf{1}$ & $\mathbf{2}$ & $\mathbf{3}$ & $\mathbf{4}$ & $\mathbf{5}$ & \\
\hline Distância mínima & 5,3 & 3,7 & 3,6 & 9,7 & 4,2 & 3,6 & 13,2 \\
Distância média & 23,6 & 27,5 & 32,8 & 31,5 & 27,6 & 19,6 & 28,6 \\
\hline Distância máxima & 44,1 & 55,2 & 58,0 & 56,2 & 55,6 & 44,1 & 50,1 \\
\hline
\end{tabular}

também um aumento da predação por insetos e outros herbívoros (Janzen, 1970).

Espécies florestais que dependem de pássaros para sua dispersão, como é o caso da canjerana, possuem alta densidade de sementes caídas sob sua copa, uma vez que os pássaros, ao se alimentarem nos galhos, promovem a sua deposição ao redor da fonte (Melo, 1997). Ferreira (1994), em suas observações, visando conhecer o comportamento das aves dispersoras de C. canjerana, concluiu que algumas espécies contribuem negativamente para a dispersão das sementes dessa espécie. Isso porque algumas espécies de aves alimentam-se somente do arilo e derrubam o resto do diásporo sob a copa, devido a limitações morfológicas, como o tamanho do bico, que impossibilitam que ele seja engolido, impedindo que essas sementes sejam dispersas corretamente.

Dentre as hipóteses de dispersão, a teoria de Escape já foi comprovada por alguns autores (Peres et al., 1997; Wenny, 2000). Essa teoria é baseada no conceito de que o pico de recrutamento de sementes ocorre a uma distância média entre a fonte e o limite de alcance dos diásporos, visando distanciar-se da mortalidade próxima a seus parentais. Entretanto, a mesma é questionada por Corrêa \& Moura (2011), pois, segundo os autores, conceitos relacionados a fatores como sítio, abertura de clareiras, dormência de sementes e hábito do dispersor também são aplicados a essa teoria. A colonização de 


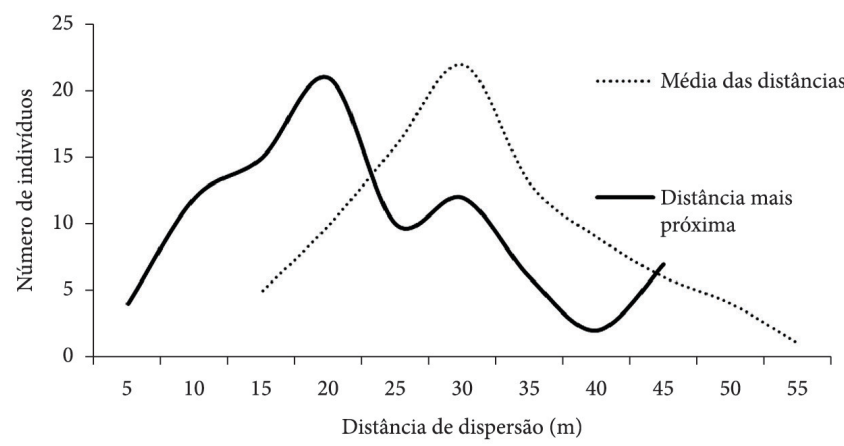

Figura 3. Frequência de indivíduos regenerantes de Cabralea canjerana encontrados na área relacionada à distância de afastamento das árvores matrizes.

Figure 3. Frequency of the regenerating individuals of the Cabralea canjerana found in the area, according to the distance spacing of the matrices trees.

sementes distantes da planta mãe pode ser relacionada à ecologia da espécie. Segundo Clark (1994), a maioria das espécies de florestas tropicais necessita de luz em alguma fase da sua vida para alcançar a maturidade reprodutiva. Logo, a procura por sítios apropriados, com pouca luminosidade na fase de regeneração, torna-se uma estratégia de espécies secundárias tardias, como no caso de C. canjerana, que necessita de luz nas fases iniciais de seu desenvolvimento mas após a emergência no dossel é capaz de desenvolver-se a pleno sol.

A distância máxima de dispersão entre um indivíduo de regeneração e sua árvore matriz mais próxima foi de 44,1 metros. A média dessa distância para todas as cinco árvores da área foi de 50,1 metros. Ao considerar que cada indivíduo regenerante é procedente da árvore matriz mais próxima, nota-se que há um pico de densidade no intervalo que varia entre 15 e 20 metros de distância (Figura 3).

Há um segundo pico de menor frequência no intervalo entre $25 \mathrm{~m}$ e $30 \mathrm{~m}$ de distância. Ao analisar a curva da distância de dispersão média, observa-se um pico único de frequência, com mais de 20 indivíduos dispersos a até 30 metros das matrizes. Há um aumento de indivíduos até esse ponto e, a partir dele, a frequência decai conforme aumenta a distância da fonte de sementes.

Essas distâncias podem estar relacionadas ao hábito dos dispersores da espécie na área. Não foram feitas observações do consumo de sementes de canjerana por pássaros na área. Contudo existem trabalhos que buscaram conhecer a fauna dispersora de C. canjerana em outras regiões do país (Ferreira, 1994; Fuzeto \&
Lomônaco, 2000; Marenzi, 2004) nos quais nota-se que a espécie é dispersa por aves comuns na região de Silveira Martins como, por exemplo, o Bem-te-vi (Pitangus sulphuratus Linnaeus, 1766), Sabiá-laranjeira (Turdus rufiventris Vieillot, 1818), Sabiá-coleira (Turdus albicolis Vieillot, 1818) (Krügel \& Behr, 2002), entre outras. Essas espécies caracterizam-se por apresentarem vôos curtos e rápidos, além de limitações morfológicas quanto ao tamanho do bico, deixando cair sementes maiores, como as de canjerana, após serem captadas (Fuzeto \& Lomônaco, 2000).

A maior frequência de indivíduos regenerantes encontra-se distante cerca de 30 metros de cada árvore matriz. Além disso, a espécie apresenta dispersão de forma agrupada na área, o que indica que alguns grupos de indivíduos tendem a se desenvolver em locais menos sombreados, gerados pelas copas das árvores adultas coespecíficas.

\section{CONCLUSÃO}

As sementes de C. canjerana têm seu pico de dispersão efetiva a uma distância média de 30 metros das árvores matrizes, sendo necessária uma árvore produtora de sementes a cada 60 metros, ou aproximadamente três indivíduos afastados por hectare a para garantir a regeneração contínua da espécie no local.

\section{STATUS DA SUBMISSÃO}

Recebido: 8 abr., 2014

Aceito: 29 out., 2014 
AUTOR(ES) PARA CORRESPONDÊNCIA

Anna Paula Zimmermann

Centro de Ciências Rurais, Universidade Federal de Santa Maria - UFSM, CEP 97105-900, Santa

Maria, RS, Brasil

e-mail: zimmermann-a@hotmail.com

\section{REFERENNCIAS}

Carvalho PER. Espécies arbóreas brasileiras. Brasília: Embrapa Informações Tecnológica; Colombo: Embrapa Florestas; 2003. Coleção Espécies Arbóreas Brasileiras 1.

Clark DA. Plant demography. In: McDade LA, Bawa KS, Hespenheide HA, Gary S. editores. La selva: ecology and natural history of a neotropical rain forest. Chicago: The University of Chicago Press; 1994.

Connell JH. On the role of natural enemies in preventing competitive exclusion in some marine animals and in rain forest trees. In: Boer PJ, Gradwell GR, editores. Dynamics of populations. Wageningen: Center for Agricultural Publishing and Documentation; 1971

Corrêa BS, Moura AS. Revisão: Relação Entre o Comportamento de Aves, a Conformação da Paisagem Fragmentada e a Estrutura das Populações de Plantas. Revista Agroambiental 2011; 3: 109-118.

Donatti CI. Consequências da defaunação na dispersão e predação de sementes e no recrutamento de plântulas da palmeira Brejaúva (Astrocaryum aculeastissium) na Mata Atlântica [dissertação]. Piracicaba. Escola Superior de Agricultura Luiz de Queiroz; 2004.

Empresa Brasileira de Pesquisa Agropecuária - EMBRAPA. Centro Nacional de Pesquisa de Solos. Sistema brasileiro de classificação de solos. Rio de Janeiro; 2006.

Ferreira EA, Souza JB, Giuaculi CP, Gomes JAJ, Paulista $\mathrm{FH}$, Oliveira CM, et al. Desenvolvimento de mudas de canjerana (Cabralea canjerana) em diferentes tipos de substrato. In: Anais da 57ª Reunião Anual da SBPC; 2005; Fortaleza, CE.

Ferreira MAP. Estudo comparado da dispersão e predação de sementes de Cabralea canjerana (Meliaceae) em duas áreas de mata do Estado de São Paulo [dissertação]. Campinas: Universidade Estadual de Campinas; 1994.

Fuzeto AP, Lomônaco C. Potencial plástico de Cabralea canjerana subsp. polytricha (Adr. Juss.) Penn. (Meliaceae) e seu papel na formação de ecótipos em área de cerrado e vereda, Uberlândia, MG. Revista Brasileira de Botânica 2000; 23: 169-176.

Hack C, Longhi SJ, Boligon AA, Murari AB, Pauleski DT. Análise fitossociológica de um fragmento de floresta estacional decidual no município de Jaguari, RS. Ciência Rural 2005; 35(5): 1083-1091. http://dx.doi.org/10.1590/ S0103-84782005000500015.

Heldwein AB, Buriol GA, Streck NA. O clima de Santa Maria. Ciência \& Ambiente 2009; 38: 43-58.

Instituto Brasileiro de Geografia e Estatística - IBGE. Classificação da vegetação brasileira, adaptada a um sistema universal. Rio de Janeiro; 2012.

Janzen DH. Herbivores and the number of tree species in tropical forests. American Naturalist 1970; 104(940): 501-528. http://dx.doi.org/10.1086/282687.

Krügel MM, Behr ER. Aves. In: Itaqui J, organizador. Quarta Colônia: inventários técnicos. Santa Maria; 2002. 256 p.

Lopes SF, Oliveira AP, Neves SB, Schiavini I. Dispersão de sementes de uruvalheira (Platypodium elegans VOG.) (Fabaceae) em um cerradão, Uberlândia-MG. Revista Árvore 2010; 34(5): 807-813. http://dx.doi.org/10.1590/ S0100-67622010000500006.

Marenzi RC. Ecologia da paisagem de um fragmento costeiro: subsídio à conservação da biodiversidade [tese]. Curitiba; Universidade Federal do Paraná; 2004.

Melo VA. Poleiros artificiais e dispersão de sementes por aves em uma área de reflorestamento, no estado de Minas Gerais [dissertação]. Viçosa: Universidade Federal de Viçosa; 1997

Nathan R, Muller-Landau HC. Spatial patterns of seed dispersal, their determinants and consequences for recruitment. Trends in Ecology \& Evolution 2000; 15(7): 278-285. http://dx.doi.org/10.1016/S0169-5347(00)018747. PMid:10856948

Peres CA, Schiesari LC, Dias-Leme CL. Vertebrate predation of Brazil-nuts (Bertholetia excelsa, Lecythidaceae), an agouti-dispersed Amazonian seed crop: a test of the escape hypothesis. Journal of Tropical Ecology 1997; 13(1): 69-79. http://dx.doi.org/10.1017/S0266467400010269.

Pizo MA. Dispersão e predação de sementes de Cabralea canjerana (Meliaceae) em duas áreas de mata do Estado de São Paulo. In: Anais do Congresso Nacional de Botânica; 1995; Ribeirão Preto, SP. p. 167.

Pizo MA, Oliveira PS. Size and lipid content of nonmyrmecochorous diaspores: effects on the interaction with litter-foraging ants in the Atlantic rain forest of Brazil. Plant Ecology 2001; 157(1): 37-52. http://dx.doi. org/10.1023/A:1013735305100.

Rabello A, Ramos FN, Hasui E. Efeito do tamanho do fragmento na dispersão de sementes de Copaíba (Copaifera langsdorfii Delf.). Biota Neotropica 2010; 10(1): 47-54. http://dx.doi.org/10.1590/S1676-06032010000100004.

Rocha VJ, Reis NR, Sekiama ML. Dieta e dispersão de sementes por Cerdocyon thous (Linnaeus) (Carnívora, Canidae) em um fragmento florestal no Paraná Brasil. 
Revista Brasileira de Zoologia 2004; 21(4): 871-876. http:// dx.doi.org/10.1590/S0101-81752004000400022.

Sork VL. Effects of predation and light on seedling establishment in Gustavia superb. Ecology 1987; 68(5): 1341-1350. http://dx.doi.org/10.2307/1939218.
Wenny DG. Seed dispersal, seed predation, and seedling recruitment of a neotropical montane tree. Ecological Monographs 2000; 70(2): 331-351. http:// dx.doi.org/10.1890/0012-9615(2000)070[0331:SDSP AS]2.0.CO;2. 